\title{
La controversia cientifico-técnica sobre las benzodiacepinas en profesionales de la salud pública de Uruguay
}

I ${ }^{1}$ Andrea Bielli, ${ }^{2}$ Pilar Bacci, ${ }^{3}$ Gabriela Bruno, ${ }^{4}$ Nancy Calisto, ${ }^{5}$ Santiago Navarro I

Resumen: La controversia científico-técnica internacional sobre las benzodiacepinas, intensa durante los años ochenta y noventa, cuestionó su lugar en la práctica clínica, por su potencialidad adictiva, y por el abuso que médicos y pacientes parecerían realizar. Este artículo presenta resultados de una investigación que tuvo como objetivo analizar el papel de dicha controversia en las prácticas médica, psiquiátrica y psicológica en los servicios de salud pública uruguayos. Se utilizó metodología cualitativa y se combinó relevamiento de artículos académicos nacionales (1960-2012), entrevistas en profundidad a 45 profesionales y dos grupos de discusión. Se efectuó análisis de contenido desde cuatro ejes: ansiedad en la clínica, prescripción, relación tratamientos farmacológicos con no farmacológicos y valoración de benzodiacepinas. Se obtuvo un panorama diacrónico de la controversia académica y se identificó una valoración condicional de estos medicamentos realizada por los profesionales que supone: reconocimiento de atributos positivos y negativos de las benzodiacepinas, uso mesurado, médicos y pacientes vigilantes de sus propios comportamientos. Se concluye que la controversia se plantea en términos individuales, lo que obstaculiza una discusión global de las dimensiones políticas y colectivas implicadas.

> Palabras-clave: benzodiacepinas; controversia científica; servicios de salud; prescripción; psicofármacos.

\author{
1 Universidad de la Republica \\ Uruguay, Facultad de Psicología. \\ Montevideo, Uruguay (abielli@ \\ psico.edu.uy). \\ 2 Universidad de la Republica \\ Uruguay, Facultad de Psicología. \\ Montevideo, Uruguay (pilarb@ \\ psico.edu.uy). \\ ${ }^{3}$ Universidad de la Republica \\ Uruguay, Facultad de Psicología. \\ Montevideo, Uruguay \\ (gbrunocamares@psico.edu.uy). \\ ${ }^{4}$ Universidad de la Republica \\ Uruguay, Facultad de Psicología. \\ Montevideo, Uruguay \\ (ncalisto@psico.edu.uy). \\ ${ }^{5}$ Universidad de la Republica \\ Uruguay, Facultad de Psicología. \\ Montevideo, Uruguay (santiago. \\ navarro.psi@gmail.com).
}




\section{Introducción}

Las benzodiacepinas (BDZ) son psicofármacos utilizados habitualmente en la terapéutica de la ansiedad y el insomnio, constituyendo sobre todo para los médicos generales un tratamiento farmacológico privilegiado para la ansiedad en sus manifestaciones agudas y crónicas.

Presentes en el mercado farmacéutico mundial desde los años sesenta, las BDZ han pasado por distintos momentos de popularidad y aceptación. Cuando hicieron aparición, se les atribuyó mayor eficacia y menores efectos secundarios que a los tranquilizantes antecesores. Durante los años setenta su consumo se incrementó en forma notoria, alcanzando gran reconocimiento de parte del cuerpo médico y del social en general como una terapéutica eficaz y segura (LADER, 1996). Sin embargo, desde los años ochenta en adelante suscitaron una controversia científico-técnica que ha puesto en cuestión su lugar en la práctica clínica médica. Dicha controversia se ha centrado en la dependencia que generan y en el abuso realizado por médicos y pacientes en sus prácticas de prescripción y consumo a largo plazo (BREGGIN, 1998; LADER; MORTON, 1991; LADER, 2011). En los países en que estos debates tuvieron lugar en el ámbito académico, profesional y mediático, las BDZ llegaron a ser vistas como un problema social y de salud pública (BURY, 1996; SCHÖNBERG, 1997).

En Uruguay las BDZ también han sido consideradas recientemente un problema de salud pública. Su elevado consumo y uso prolongado más allá de los plazos usualmente recomendados constituyen las principales dimensiones del problema, el cual se ha intentado explicar aludiendo a la baja percepción de los riesgos por parte de los médicos y su uso en indicaciones de eficacia no comprobada (SPERANZA et al., 2015).

En este artículo nos centraremos en las características particulares que la controversia científica sobre las BDZ ha tomado en Uruguay y en cuáles han sido sus consecuencias clínicas. Específicamente analizaremos el papel de dicha controversia en el uso que se hace de ellas en las prácticas de la medicina general, la psiquiatría y la psicología en los servicios de salud pública de Montevideo. Para ello, primero se presenta y caracteriza la controversia internacional sobre las BDZ. En segundo lugar, se describe el desarrollo de la discusión sobre ellas en el medio académico uruguayo de 1960 a 2012. En tercer lugar, se identifica 
y discute el estado actual de las valoraciones, argumentaciones y actitudes de

los profesionales de la salud. Por último, se presenta un panorama de problemas emergentes en la clínica percibidos por estos profesionales a partir su uso.

\section{Metodología}

Es una investigación exploratoria de diseño cualitativo que combinó una revisión documental de publicaciones del ámbito académico uruguayo sobre el tópico de la investigación y la realización de entrevistas y grupos de discusión con profesionales de la salud pública de Montevideo. El trabajo de campo se realizó durante los años 2013 y 2014.

Para la revisión documental se hizo una búsqueda de artículos nacionales de autores uruguayos, no publicados en el extranjero, ubicados en revistas que no se encuentran indexadas, en repositorios de bibliotecas de la Universidad de la República y de asociaciones científicas del país. El relevamiento documental local comprendió artículos académicos sobre BDZ y ansiedad a partir de 1990 hasta el año 2012. Se tomó como punto de partida el año 1990 ya que desde entonces fue notoria la pérdida de popularidad de las BDZ en Europa y Estados Unidos. Posteriormente, dado que el volumen de artículos relevados lo permitía, el período fue extendido hasta el año 1960, abarcando así un panorama de las discusiones académicas en el medio desde la llegada de las BDZ al país. Las fuentes relevadas fueron las siguientes: Revista de Psiquiatría del Uruguay, Revista Médica del Uruguay, Archivos de Medicina Interna, Diario Médico, Tendencias en Medicina y Cursos de Perfeccionamiento de Psiquiatría.

Para la sistematización de las publicaciones se confeccionó una grilla con los siguientes ítems: datos de identificación del artículo (revista, año, autor, título, páginas), institución a la que pertenece el autor, biblioteca en la cual se encuentra, tipo de artículo, tema principal y aportes.

Para el análisis documental, se categorizó la información de acuerdo a tópicos claves en el uso clínico de las BDZ: conceptualización de la ansiedad utilizada, información científica sobre las BDZ, recomendaciones para su uso, valoración de su utilidad clínica, valoración de las prácticas de prescripción, ventajas y desventajas de las BDZ, percepción de su uso y/o prescripción como problema de salud pública, tipo de uso asociado a la ansiedad, tipo de uso asociado a trastornos 
del sueño, cambios en las indicaciones asociadas a estos medicamentos. Asimismo, estos artículos fueron se ingresaron al programa Atlas.ti 6.1 para triangular la información con el análisis de las entrevistas y los grupos de discusión.

Las técnicas de entrevista en profundidad y grupos de discusión permitieron identificar opiniones y percepciones de los participantes sobre el uso de BDZ en relación a su práctica profesional, así como determinar discrepancias, consensos $\mathrm{y}$ tensiones.

Primeramente se seleccionaron los Centros de Salud donde llevar a cabo el trabajo de campo cubriendo diferentes zonas geográficas de Montevideo. Luego se contactó a los profesionales por teléfono o directamente, concurriendo a los lugares de trabajo. Se buscó entrevistar médicos generales y de familia, psiquiatras y psicólogos contratados de forma estable por en el sector público de la salud, con no menos de tres años de ejercicio de la profesión, insertos en el primer y segundo nivel de atención, en los que son frecuentes las consultas vinculadas a ansiedad e insomnio. Se procuró que trabajasen en diversas unidades asistenciales (Hospitales, Equipos de Salud Mental, Centros de Salud, Policlínicas) y cumplieran diferentes funciones dentro de las mismas (directores, jefes de servicio, profesionales con atención directa). La muestra se segmentó por profesiones, según la proporción de las mismas dentro de los recursos humanos de los servicios públicos. Por consiguiente, se realizó un mayor número de entrevista a médicos generales y de fimilia, luego psiquiatras y por último psicólogos. Los psicólogos fueron incluidos por constituir un grupo profesional en contacto e intercambio con médicos y psiquiatras en el abordaje clínico de la ansiedad. En suma, se entrevistaron ocho médicos generales, diez médicos de familia, once psiquiatras y seis psicólogos, totalizando 35 entrevistas. La cantidad de entrevistas realizadas se justificó en el criterio de saturación teórica. Todas las entrevistas se realizaron en el lugar de trabajo de los participantes, y duraron en promedio 45 minutos, 33 fueron grabadas y dos registradas por escrito.

Paralelamente, se entrevistaron diez informantes calificados que, por su trabajo académico en materia de prescripción y consumo de BDZ y conocimientos en el campo de la salud pública, aportaron elementos sustanciales sobre el estado de la controversia de las BDZ en Uruguay. Siete provenían del campo de la medicina, dos de las ciencias sociales y uno de otra profesión del área de la salud. 
Las entrevistas realizadas a médicos generales, de familia y psiquiatras incluían en su pauta cuatro bloques: ansiedad en la clínica, prescripción de BDZ, relación con otros tratamientos farmacológicos y no farmacológicos y percepción acerca de las BDZ. Para las entrevistas con psicólogos se eliminó el bloque sobre la relación de las $\mathrm{BDZ}$ con otros tratamientos por considerarlo pertinente sólo para la práctica médica. Para los informantes calificados la pauta de la entrevista incluía tres apartados: debate sobre las BDZ en Uruguay, prescripción de BDZ y un bloque que se adecuaba al perfil del profesional.

Veamos algunos ejes que nos permiten caracterizar la población de profesionales que participaron en las entrevistas en profundidad. La figura 1 muestra la distribución de dichos profesionales de acuerdo a grupos etarios:

Figura 1. Distribución de los profesionales por grupos etarios

\begin{tabular}{|l|l|l|l|}
\hline Profesión & Edad & \multicolumn{2}{|l|}{} \\
\hline & 30 a 39 años & 40 a 49 años & 50 a 59 años \\
\hline Médicos generales & 2 & 3 & 2 \\
\hline Médicos de familia & 1 & 4 & 3 \\
\hline Médicos Psiquiatras & & 4 & 6 \\
\hline Psicólogos & & 4 & 2 \\
\hline Total & 3 & 15 & 13 \\
\hline
\end{tabular}

\begin{tabular}{|c|c|c|c|c|}
\hline \multirow{2}{*}{ Edad } & & & & \\
& & 30 a 39 años & & \\
Médicos generales \\
Médicos de familia \\
Médicos Psiquiatras \\
Psicólogos
\end{tabular}


La figura 2 muestra la distribución por rangos de experiencia en el ejercicio de la profesión.

Figura 2. Distribución de profesionales por años de ejercicio de la profesión

\begin{tabular}{|l|l|l|}
\hline Profesión & Años ejercicio & \multicolumn{2}{|l|}{} \\
\hline & menos de 10 años & de 10 a 20 años \\
\hline Médicos generales & & 4 \\
\hline Médicos de familia & 3 & 2 \\
\hline Médicos psiquiatras & 2 & 3 \\
\hline Psicólogos & & 2 \\
\hline Total & 5 & 11 \\
\hline
\end{tabular}

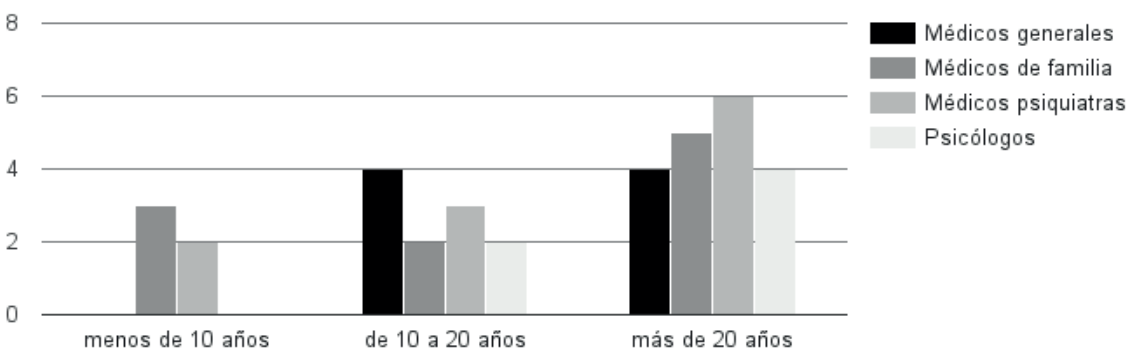

En cuanto a la distribución por sexo, los entrevistados en su gran mayoría fueron mujeres. Esto se debió principalmente a dos razones: en primer lugar, a la feminización del plantel profesional de los servicios de salud especialmente de médicos de familia y psicólogos; en segundo lugar, las mujeres fueron las más dispuestas a participar en la investigación.

Se realizaron dos grupos de discusión, uno conformado por seis médicos generales y médicos de familia y otro por cuatro psicólogos. El grupo de psiquiatras no pudo concretarse, asistieron dos profesionales, cuyas opiniones fueron integradas al análisis de las entrevistas.

Para los grupos de discusión se elaboraron dos pautas de trabajo (una para médicos generales, de familia y psiquiatras y otra para psicólogos) tenían 
como disparador recientes artículos de prensa nacionales acerca del tema y que

cubrían los tópicos de ansiedad en la clínica, prescripción de BDZ y percepción sobre las BDZ.

Con la información obtenida se efectuó un análisis de contenido (programa Atlas.ti 6.1) del material recogido en las entrevistas y en los grupos de discusión desde cuatro ejes: ansiedad en la práctica clínica, acto de prescripción, relación de tratamientos farmacológicos con tratamientos no farmacológicos y valoración de los ansiolíticos benzodiacepinicos. También se procedió a la realización de un mapeo de las oposiciones emergentes en las entrevistas y sus argumentos de base, a la identificación de las metáforas utilizadas por los entrevistados en sus narrativas, y se elaboró un cuadro de síntesis de las respuestas dadas por cada participante a las preguntas de investigación.

Los artículos académicos se analizaron utilizando categorías clave en el uso clínico de las BDZ: conceptualización de la ansiedad utilizada, información científica sobre las BDZ, recomendaciones para su uso, valoración de su utilidad clínica, valoración de las prácticas de prescripción, ventajas y desventajas de las $\mathrm{BDZ}$, percepción de su uso y/o prescripción como problema de salud pública, tipo de uso asociado a la ansiedad, tipo de uso asociado a trastornos del sueńo, cambios en las indicaciones asociadas a estos medicamentos. Asimismo, estos artículos fueron ingresados al programa Atlas.ti 6.1 para triangular su análisis con el análisis de las entrevistas y los grupos de discusión.

El presente estudio fue avalado por el Comité de Ética en Investigación de la Facultad de Psicología de la Universidad de la República regulado a partir del Decreto Ley del Poder Ejecutivo de la República Oriental del Uruguay CM/515 - 04/08/2008 que se establece en conformidad a lo dispuesto en la Declaración Universal de Derechos Humanos, Declaración de Helsinki y la Declaración Universal sobre bioética y Derechos Humanos.

\section{Las controversias científicas y la controversia sobre BDZ}

Las controversias científico-tecnológicas representan una parte sustancial y constitutiva del proceso de producción del conocimiento científico, y su estudio permite acercarse a la ciencia tal cual es desarrollada por sus protagonistas (AIBAR, 2002; MEYER, 2009). Proporcionan una visión dinámica de la ciencia, en la que ésta ya no es entendida como una empresa estrictamente 
racional y sólidamente unificada, sino como una actividad en la que dimensiones no-racionales, políticas y sociales, internas y externas a la comunidad académica, hacen de ella una empresa en movimiento y en tensión permanente (BRANTE; ELZINGA, 1990). Toda controversia científica supone la existencia de opiniones y argumentos contrapuestos relativos al conocimiento, una contradicción manifiesta que divide y antagoniza a los colectivos pero que a su vez los pone en interacción y los impulsa al diálogo (BRANTE; ELZINGA, 1990). Las controversias pueden tener lugar únicamente en el ámbito académico o alcanzar además el ámbito público, llegar a una clausura o permanecer abiertas a lo largo del tiempo (MEYER, 2009).

Los debates sobre las BDZ constituyen un ejemplo reciente de una controversia científico-técnica desarrollada en el campo médico. Un primer momento de controversia tuvo lugar en los años ochenta, cuando en países como Estados Unidos, Alemania y Gran Bretaña, las discusiones sobre sus ventajas y desventajas incidieron directamente en la caída de sus ventas (BURY, 1996; LADER, 1996). Los debates se centraron principalmente en torno a la capacidad de las BDZ de generar farmacodependencia, inducida además por el propio sistema de salud y sin el conocimiento de los pacientes (SOLAL, 1994), al uso inapropiado que médicos y usuarios hacían de ellas y la trivialidad de los problemas de salud para los que eran utilizadas. El cuerpo médico se dividió entre aquellos que sostenían que las BDZ eran excelentes medicamentos ansiolíticos e hipnóticos y aquellos que ponían en cuestión su utilidad clínica. Se cuestionó fundamentalmente la naturaleza de esta dependencia: jera posible hablar de dependencia aunque no hubiera incremento en las dosis y se utilizarán las dosis terapéuticas? ¿la dependencia se producía en todos los casos o sólo en aquellos que presentaban una predisposición? ¿la dependencia se debía a las características del medicamento o al uso prolongado que hacían los pacientes? (LADER, 1978). Paralelamente, se estableció una fuerte tensión entre médicos generales y psiquiatras debido a la crítica de estos últimos sobre las prácticas de prescripción de los primeros. Estas críticas responsabilizaban a los médicos generales por prescribir BDZ indiscriminadamente sin advertir a los pacientes sobre los riesgos de dependencia y sin ayudarlos luego a abandonarlas (BURY, 1996).

En Gran Bretaña y Suecia, en donde las discusiones alcanzaron la arena pública, los medios masivos de comunicación promovieron el debate fundamentalmente 
en torno a la conversión involuntaria de los pacientes en adictos (BURY, 1996; LADER, 1996; SCHÖNBERG, 1997). Esto permitió el involucramiento de grupos de activistas que utilizaron el argumento de la adicción involuntaria para impulsar litigios contra médicos y servicios de salud. En los medios de comunicación suecos, por ejemplo, la responsabilidad sobre la dependencia se trasladaba de los usuarios a los médicos y de estos al sistema de salud. A través de testimonios de usuarios, se presentaban interpretaciones diversas de lo que significaba la dependencia las BDZ y el alcance y la variedad de síntomas que indicaban la presencia de este problema. Esta campaña mediática recibió duras críticas de un grupo de médicos que definía el problema como causado por las prácticas descuidadas de algunos pocos médicos y las prácticas abusivas de un número menor de usuarios (SCHÖNBERG, 1997, p. 21-22). Una posición similar sostuvo la American Psychiatric Association que en 1990 publicó el resultado un grupo de trabajo sobre las $\mathrm{BDZ}$ en el que se concluía que dichos fármacos son medicamentos seguros de leves efectos secundarios cuando se utilizan de manera apropiada bajo la supervisión médica (SALZMAN; SHADER, 2015).

Actualmente las BDZ continúan siendo un tratamiento farmacológico controvertido. Las dudas acerca de su utilidad clínica persisten e incluso recientemente se las ha asociado con el desarrollo de Alzheimer (DONOGHUE; LADER, 2010; SALZMAN; SHADER, 2015). Su uso extendido en el primer nivel de atención en salud permanece como una preocupación para los servicios de salud (LADER; TYLEE; DONOGHUE, 2009), sin embargo algunos sostienen que el debate sobre las $\mathrm{BDZ}$ ha generado una contraproducente reticencia a su uso tanto en médicos como en pacientes, configurando una situación de subutilización de un medicamento eficaz y seguro (SALZMAN; SHADER, 2015). Asimismo, otros señalan que esta controversia ha promovido la suplantación de las BDZ por los antidepresivos en el tratamiento de la ansiedad sin contar con evidencia científica sólida que respalde este cambio (BALON, 2013). En suma, las BDZ constituyen aún hoy uno de los grupos de psicofármacos más utilizados en el tratamiento de la ansiedad y el insomnio (STAHL, 2002) y continúan generando posiciones antagónicas dentro del cuerpo médico que en los últimos tiempos no sólo ha debatido sobre sus ventajas y desventajas como terapéutica sino que también ha debatido sobre la propia controversia de las BDZ. Esta controversia se da en el contexto de una inflación diagnóstica que ha acarreado un uso 
excesivo de medicamentos psiquiátricos beneficiando a la industria farmacéutica, aumentando los ingresos económicos. El uso de fármacos "milagrosos" incluyó las BDZ, que fueron un éxito por provocar menos efectos secundarios y permitir una fácil prescripción en el ámbito de la atención primaria, lo que amplió el uso de medicación psicofarmacológica en la población sana (FRANCES, 2014).

\section{La discusión sobre las BDZ en el medio académico uruguayo}

El debate sobre las BDZ en el Uruguay no fue un debate mediático ni fuertemente interactivo, sino que se desarrolló de manera paulatina en el seno del medio académico nacional. Es posible distinguir cuatro fases en el tratamiento dado a las BDZ por la medicina uruguaya: 1) un momento de recepción optimista, 2) un momento de consolidación de las BDZ como tratamiento de la ansiedad, 3) un momento de surgimiento de la prescripción de las $\mathrm{BDZ}$ como problema 4) y un momento de abandono de las $\mathrm{BDZ}$ como tratamiento principal de la ansiedad.

1) momento de recepción de las BDZ (años 60): en esta fase las BDZ son recibidas por la medicina uruguaya con optimismo. Se realizan las primeras experiencias clínicas con $\mathrm{BDZ}$ en el país principalmente por docentes de la Facultad de Medicina e impulsadas por el Laboratorio Roche, en algunos casos incluso antes de que la BDZ ensayada estuviera disponible en los mercados. Se estudian sus perfiles, sus ventajas y desventajas en comparación con el meprobamato y los barbitúricos, sustancias que hasta el momento se utilizaban como tranquilizantes. Se ensayan el clordiazepóxido (GOLDSTEIN, 1966), el diazepam (GALEANO MUÑOZ; BEDÓ, 1961; GOLDSTEIN, 1963a, 1963b; FERRARI FORCADE; ALFONSO TRINDADE, 1963), el medazepam (GALEANO MUÑOZ, 1969; GOLDSTEIN, 1969), el nitrazepam (GALEANO MUÑOZ; ULRIKSEN DE VIÑAR; VIÑAR, 1964; REY, 1964). La valoración general que se realiza de las ellas es muy positiva, se insiste en que producen efectos secundarios mínimos.

2) momento de consolidación de la asociación entre BDZ y ansiedad (años 70 y 80 ): en esta etapa toma cuerpo la noción de la existencia de una psicofarmacología de la ansiedad, es decir, un tratamiento definido para los estados ansiosos apoyado fundamentalmente en las BDZ. Desde finales de 
los años 70 aparecen menciones a las desventajas de las BDZ, con referencias

al problema de la tolerancia, la dependencia y las reacciones de abstinencia, pero estas desventajas no son enfatizadas como un obstáculo mayor para su uso (GASPAR, 1978; HERRERA, 1978; MURGUÍA, 1987).

3) emergencia de la prescripción de BDZ como problema (años 90): en esta fase aparecen estudios puntuales sobre las prácticas de prescripción de los médicos uruguayos en los que se problematiza el uso extendido de las $\mathrm{BDZ}$ a nivel de grupos poblacionales específicos (preferentemente mujeres a partir de los treinta años) y la prescripción realizada mayoritariamente por médicos generales (REY, 1998). Se realiza también un primer estudio sobre consumo, que aunque limitado en su alcance, señala una cierta tendencia a un alto consumo en el país (BUSTELO, 1994).

4) abandono de las BDZ como terapéutica de primera elección (del 2000 en adelante): en esta etapa aparecen recomendaciones precisas sobre su uso por tiempos limitados y son los antidepresivos ISRS los que pasan a ser el tratamiento de primera elección para los trastornos de ansiedad, en la medida en que se los considera más eficaces que las $\mathrm{BDZ}$ en la reducción de los síntomas psíquicos de la ansiedad, poseen la ventaja de tratar con un mismo medicamento los casos de comorbilidad de ansiedad y depresión, y detentan un perfil de efectos secundarios que son tolerados sin mayores dificultades (BOUSSARD et al., 2002). Asimismo, hacia el año 2010 aparece claramente la preocupación por el uso racional de estos medicamentos (DANZA; CRISTIANI; TAMOSIUNAS, 2009; MATO et al., 2012).

La controversia de las BDZ en Uruguay adquirió la forma de un proceso de viraje de distintas visiones sobre las BDZ a lo largo del tiempo. Comenzó con una visión positiva de las mismas que se fue empeñando en la medida en que, en consonancia con los debates internacionales, empiezan a señalarse algunas de sus desventajas. Luego se suman advertencias sobre el uso de las mismas en la clínica hasta que llega a recomendarse su empleo limitado y a veces su suplantación por otros tratamientos farmacológicos. En los últimos años los artículos académicos coinciden en recomendar su uso por tiempos no mayores a cuatro semanas, es decir, evitando el uso crónico, con dosis flexibles y disminución gradual. Sin embargo, se identifican también la existencia de algunos tópicos tensionantes que 
coinciden con aquellos en torno a los cuales se desarrolló la controversia sobre las $\mathrm{BDZ}$ a nivel internacional: la tensión adicción-dependencia, banalización del uso y consumo excesivo, la responsabilidad de médicos generales en los usos a largo plazo, la presión de los pacientes para conseguirlas.

\section{Valoraciones, argumentaciones y actitudes actuales de los profesionales de la salud}

A través de las entrevistas y grupos de discusión con los profesionales de la salud fue posible obtener un panorama actual de sus valoraciones sobre las BDZ, de las argumentaciones a las que apelan para fundamentar su uso en la práctica clínica y de las actitudes favorables o negativas hacia estos medicamentos.

La valoración global que médicos generales, de familia, psiquiatras y psicólogos realizan sobre las $\mathrm{BDZ}$ se sustenta en la enumeración de una serie de ventajas y desventajas asociadas a las mismas que configuran significaciones tanto positivas como negativas sobre las BDZ.

Los atributos positivos nombrados por los entrevistados coinciden a grandes rasgos con los mencionados por la literatura internacional al caracterizar perfil de acción: son medicamentos que reducen la ansiedad, son seguros pues los pacientes no logran quitarse la vida con ellos, son rápidamente efectivos, incluso se las llega a comparar con los barbitúricos si bien estos ya no se utilizan en la práctica clínica actual. Los atributos negativos mencionados coinciden con los efectos secundarios usualmente reconocidos en las BDZ: riesgos de intoxicación, especialmente con el diazepam, problemas de memoria, caídas, tolerancia y sobre todo su potencial adictivo, dependencia con su consiguiente síndrome de abstinencia. Las significaciones positivas y negativas ligadas a estos atributos dificultan la existencia de una interpretación consensual sobre las $\mathrm{BDZ}$ entre los profesionales y ante todo deja en evidencia la existencia disensos en torno a su seguridad.

Por ejemplo, un médico general expresaba: "como que han pasado, no es que hayan pasado a un segundo plano pero, pero no las considero muy seguras. Eh, son seguras, son seguras entre comillas (GMG1)". Mientras que otro médico general afirmaba: "Tiene también un margen de seguridad importante, nosotros siempre decimos, estee, si un paciente tiene un intento de autoeliminación, nosotros siempre decimos que con benzodiacepinas puede tomarse el blíster entero que lo que le va a pasar es que va a dormir mucho" (GMG4). 
Este disenso también aparece en torno a la evaluación de los profesionales sobre los beneficios que las BDZ les brindan a los pacientes y por consiguiente, sobre los riesgos y beneficios que el acto mismo de prescripción implica. Los beneficios que se mencionan se relacionan a la mejoría de los síntomas y de la calidad de vida del paciente. Esta última, se lograría en la medida en que las BDZ, ayudarían a solucionar "problemas familiares" (NMF2) y restablecerían en los pacientes la capacidad de "funcionar". Para los psicólogos, por ejemplo, las BDZ también serían útiles para posibilitar los tratamientos psicoterapéuticos: "yo creo que la medicación a veces puede, puede ayudar en algo, pero creo que no tiene que ser lo único en la vida, ta. A veces ayuda, sí, porque si... yo que sé, hay cosas que no las podés trabajar en la medida que vos no estés más tranquilo” (AnPSIC1).

Sin embargo, al mismo tiempo, las BDZ enfrentarían al paciente a riesgos concretos que hacen de la prescripción un acto paradójico:

Si no iniciás el tratamiento corrés riesgo y si lo iniciás también, porque muchas veces,
a mí me ha pasado, este, que iniciás algún tipo de ansiolítico... y el paciente, yo le
explico: "mirá, es un cambio en toda la forma de vivir, la pastilla te va a ayudar en algo
pero empezá con cuarto comprimido, pero si no te hace efecto no subas a uno o a dos
por tu cuenta". Sin embargo, hay gente que se agarra el blíster, como se tomó uno y
se siguió sintiendo igual pum, pum, pum, se tomó tres o cuatro y después vienen con
una carta del Hospital Psiquiátrico, 'paciente que hizo un intento de autoeliminación',
cuando ellos te dicen que no se trataron de matar, se trataron de dormir (PPSIQ4).

Esta paradoja de la prescripción se refleja en una valoración global de las BDZ también paradójica, que hace que sean definidas por algunos como "excelentes fármacos" (AlPSIQ2) y por otros como "una medicación que no es buena” (NMF1). En torno a dichas valoraciones contrapuestas, se despliegan también actitudes antagónicas ante el uso de las BDZ en la clínica. Algunos profesionales muestran una clara reticencia a recetar $\mathrm{BDZ}$ y declaran tratar de evitar la prescripción. La prescripción los implica como sujetos, produciendo reacciones afectivas negativas: "trato de... recetar lo menos posible porque no, no me gustan" (NMF1, AnMG1, AnPSIQ2), "nos genera un poco de, de ansiedad a nosotros vamos a decir" (NMF2), "Cada vez me cuesta más" (GMF1), "indico cuando estoy muy segura” (GMF2), "son riesgosas, les tengo miedo" (AlMF2). Estas emociones no impiden que la prescripción tenga lugar, pero ésta se fundamenta en una serie de argumentos que incluyen aspectos de la presentación clínica de los pacientes (intensidad de los síntomas, agudeza) y por sobre todo argumentos que hacen referencia a la falta de alternativas, ya sea medicamentosas o de otro tipo. 
Se utilizan expresiones como "a veces no, no nos queda más remedio un poco por la, por las situaciones que nos plantean" (NMF2, NPSIQ2, GMF1), "no tenés otra, muchas veces no tenés otra" (AnMG1), "algo le tenemos que dar" (NMG1).

Un psiquiatra, resaltaba por ejemplo la inevitabilidad del uso de las BDZ al mismo tiempo que indicaba los recaudos que son necesarios para ello:

[...] uno no puede dejar un paciente con la sensación de que se puede matar o que pueda ser una situación mucho más grave de lo que está ocurriendo y, entonces, a veces, bueno, el uso de benzodiacepinas es obligatorio, pero intento que sean tiempos cortos y que el paciente tenga conciencia de que es de riesgo (PPSIQ 4).

Las diferentes valoraciones acerca de las ventajas o desventajas de las BDZ, así como con respecto a su seguridad o los efectos adversos graves pero no letales, evidencian que el tema que nos ocupa continúa siendo objeto de controversia, por lo menos a nivel de la práctica profesional. Asimismo es a destacar que los entrevistados muestran diferentes visiones acerca de la seguridad del fármaco, cuestión que luego se desplazará del medicamento al sujeto que lo consume o al médico que lo prescribe de manera inadecuada.

El uso que se hace de las $\mathrm{BDZ}$ se vuelve el rasgo esencial por el que se define la valoración del medicamento. Es el buen uso o el mal uso que hacen los profesionales y los pacientes de las $\mathrm{BDZ}$ los que definen su carácter positivo o negativo, ya que la $\mathrm{BDZ}$ en sí misma sería indiferente:

Y yo creo que nada es bueno ni malo, es cuando hablamos de alimentación, la naranja no es ni buena ni mala, depende qué hagas con la naranja. Esto es lo mismo, con los huevos, o la carne o la grasa, qué sé yo, es lo mismo. Un medicamento no es ni bueno ni malo en sí mismo, depende cómo lo usamos (AlMF1).

$\mathrm{O}$, en todo caso, un buen medicamento que deja de serlo únicamente por responsabilidad de los sujetos implicados: "Pero son excelentes, el tema es el manejo que se hace de las benzodiacepinas, no es la droga la que, es el manejo" (PPSIQ4).

Surgen entonces diversas caracterizaciones y valoraciones morales del comportamiento de unos y otros, de quienes se espera un control sobre sí mismos en relación al uso que hacen del medicamento. El profesional debe, por consiguiente, lograr ciertos controles sobre los comportamientos de los pacientes, por ejemplo, "hacer que el paciente venga a la consulta" (GMF2), pero también lograr ciertos controles sobre sí mismo: "indicarlas con criterio" (GMG1), "cierta conciencia de que lo tengo que sacar lo antes posible" (AIPSIQ1), "prescribirlas con cuidado" (PPSIQ2, Grupo MF). 
Actos que implican un comportamiento responsable:

[...] o sea la medicación tiene que pensarse, o sea, realizar la medicación tiene que pensarse mucho, tiene que ser meditado ¿̨no? O sea tiene, tiene, el médico tiene que ser responsable de lo que está haciendo porque sino está transformando la población en je, je, je, seres adictos ¿no? (NMF2).

Los pacientes son caracterizados por una serie de comportamientos inadecuados, que desafían la autoridad médica y que inevitablemente les conduce a un mal manejo de la medicación. El paciente va a terminar tomando más de lo que el médico indicó o tomando el medicamento sin indicación médica, en usos no que no son para la ansiedad y resultan injustificados: "por las dudas que me duela" (GMF3). Se utilizan expresiones como "la gente se automedica" (AnMG1, Grupo MF), "la gente toma cantidades, mezcla dos, tres benzodiacepinas" (AnMF1), "el paciente abusa" (AlMG1).

Esta moralización de los comportamientos de los pacientes, se acompaña de una culpabilización de los mismos:

Usted abre la puerta y el ladrón entra, ¿quién es el culpable, el que le abrió la puerta o el ladrón que entró?, en realidad, es más responsable el ladrón, porque el ladrón entró, pero vio la oportunidad. Entonces, el tema es que uno indica una benzodiacepina y luego el paciente hace un uso de la benzodiacepina apropiado o no apropiado, eso queda en el secreto del consultorio (PPSIQ4).

Esto se traduce en un conjunto de tensiones en la relación médico-paciente expresadas en la caracterización que se realiza de los mismos como pacientes que resisten a las sugerencias del profesional, que actúan con independencia o incluso se equivocan al seguir las indicaciones de los médicos. También emergen en los relatos que describen a los pacientes utilizando fuentes no expertas para la obtención de información y conocimiento sobre el medicamento y desplegando estrategias de acceso al medicamento por fuera del sistema de salud.

Claro, son medicamentos que son necesarios, digo, vamos a entendernos, que son buenos, o sea, bien usados. El tema, que yo pienso, actualmente es que están sobre-usados, demasiado usados, y que la gente a su vez se automedica también. Porque además, yo que sé, si no lo consiguen con una receta van y lo consiguen en la feria, digo, es una cosa, no sé, ahora parece que se ha regulado eso, no lo sé, no he ido por la feria (AnMG1).

Aún así, la percepción de las BDZ como un problema de salud pública genera posiciones contrapuestas. Se considera tanto como un tema perimido, ya solucionado por la aparición de medicamentos como los antidepresivos ISRS 
que al también poseer indicación para el tratamiento de la ansiedad pueden ser utilizados en lugar de estas, como un tema grave y actual por la sospecha de un uso de las $\mathrm{BDZ}$ en el país que no se ajusta a los consensos y recomendaciones internacionales que sugieren utilizarlas con dosis flexibles y por períodos no mayores a las ocho semanas.

Una psiquiatra entrevistada, por ejemplo, expresaba: "Si estará demodé el tema, que en el mercado ya no está la benzodiacepina. Se está buscando alternativas y se está buscando otro tipo de fármacos. La guerra de las benzodiacepinas terminó." (NPSIQ1). Mientras otra psiquiatra sostenía:

No estoy en contra de las benzodiacepinas, me parece un fármaco que, simplemente, eh, como tiene, hay una cuestión cultural de un uso abusivo [...] Es una cuestión del riesgo que tienen las benzodiacepinas, en nuestro, en el Uruguay de hoy, de un mal uso. Ahí yo veo el riesgo mayor, el mal uso de las benzodiacepinas. No es que no tengan nunca indicación, no, me parece que eso es una exageración (PPSIQ1).

El problema se define por la percepción de un alto uso de BDZ en el país (PPSIQ2, PMF1, AlMF1, AlMF2), y como por algunas prácticas cuestionables: distintos especialistas recetando BDZ a los pacientes, la inclusión regular de $\mathrm{BDZ}$ en el conjunto de medicamentos que se le prescriben al paciente "como un tiro de gracia” (NMF2). La situación se describe como un "abuso", se la cataloga de "descomunal” (PPSIQ1, PMG1, AlMG1, AlPSIC1, NMG1), se considera que los demás profesionales realizan un uso indiscriminado de las BDZ (AlPSIQ1), sobremedican (PPSIC1), sobreutilizan (AIPSIQ 2), "inundan" de BDZ a los pacientes (NMG2), dopan pacientes (AnPSIC1), y "los medicamentos se tratan a la ligera" (AnMF1 ). En términos generales, el problema se define como un exceso, ya sea del médico al recetar muchas, o del paciente al consumir mucho. Y donde los médicos parecen no tener conciencia de lo que están generando:

El tema es que no hay una conciencia real, una conciencia mórbida de lo que estamos generando. La hay en los últimos tiempos, pero los psiquiatras cada vez medicamos menos y los médicos generales, cada vez medican más. Pero tú tenés hipertensos que vienen a repetir, entonces, están tomando conmigo tal cosa y tal cosa y con el médico general, diazepam. Yo caigo muerta con el cardiólogo, no puedo creer. Porque, de repente, a veces, toma una benzodiacepina que yo le indico más diazepam que le indica el cardiólogo. Es rarísimo (PPSIQ4).

Es claro que, en las opiniones de los entrevistados, encontramos consideraciones críticas acerca de la modalidad de prescripción y el uso de las BDZ que reflejan el consumo excesivo de estos fármacos como un problema de salud pública. Esta 
perspectiva coincide con las afirmaciones realizadas desde la academia por el estudio de Speranza et al. (2015).

\section{Problemas emergentes en la clínica a partir del uso de las BDZ}

Como telón de fondo a las valoraciones sobre las BDZ, aparece un conjunto de situaciones problema para la práctica clínica en torno a su uso: retiro de medicación en pacientes mayores, dificultades en el manejo de repetición de la medicación, dificultades para la derivación a especialistas.

Por ejemplo, si bien se expresa que se intenta no repetir la medicación sin indagar la pertinencia de la indicación, se presentan una serie de argumentos que harán la excepción a la regla, por lo cual el médico deberá repetir aún en contra de su pensamiento. Por ejemplo, cuando el paciente no continuará atendiéndose con él, cuando indicación viene dada previamente por un especialista, cuando tienen patologías particulares.

Los pacientes mayores representan una dificultad clínica como consumidores crónicos de BDZ. La actitud de estos pacientes es identificada como un escollo, dado que se resisten a dejar la medicación que toman desde hace años (NMF1, PPSIQ2, Grupo MF) y le llegan al profesional medicados por otros. Al mismo tiempo se esboza la idea de que no vale la pena sacarle el medicamento a los pacientes mayores porque les complejizaría su vida cotidiana (AnMG1, AlMF1, AnPSIQ2, AnPSIQ3, PPSIC1, PPSIQ3) y porque además cuesta mucho trabajo al médico (AnMF1, Grupo MF). "Acá son pacientes que hace treinta años que están tomando, y le vas a sacar los dos o tres Diazepam o Lorazepam por día, no, ¿para qué?, les va a quedar un malambo que no vale la pena.”. (AnPSIQ3)

Al mismo tiempo, algunas características del funcionamiento de los servicios de salud impulsarían el uso de las BDZ. En este sentido, se verifica un uso de las BDZ mientras no puede derivarse al paciente al equipo de Salud Mental o cuando aún habiéndose hecho la derivación el paciente no ha consultado al especialista. Esto explica tanto la decisión de iniciar un tratamiento como la decisión de mantenerlo. Sobre todo por médicos generales y médicos de familia que expresan tomar a su cargo casos que deberían ser tratados por el especialista, dadas las dificultades para acceder a ellos, ya sea por cuestiones institucionales o por el costo de terapias psicológicas. Aparece la noción de que deben "contener" al 
paciente (AnMG1, Grupo MF), de que los médicos generales y de familia "hacen la terapia” que deberían hacer los especialistas en Salud Mental (Grupo MF).

Estas situaciones hacen peligrar los recaudos que los profesionales deberían tomar a la hora de la prescripción.

\section{Conclusiones}

La controversia sobre las BDZ ha tenido variadas expresiones en los diferentes ámbitos locales. En el presente estudio se pudo establecer para el Uruguay la existencia de una valoración controversial del uso clínico de las BDZ que adopta diferentes formas a lo largo del tiempo. A pesar de la inexistencia, de una controversia franca ni en el medio académico nacional ni en el ámbito público, se identifica una variación de la percepción de la valoración de las BDZ a lo largo de las décadas que ha variado desde considerarlas en los años sesenta medicamentos más ventajosos y más seguros que sus antecesores a considerarlas actualmente como medicamentos que deben utilizarse con mesura. Asimismo, las consideraciones que se realizan sobre las BDZ presentan argumentos similares a los utilizados en controversias internacionales especialmente en torno a los hábitos de prescripción de los médicos generales y en torno a la predisposición de algunos pacientes a volverse adictos a estos medicamentos.

Actualmente, la valoración general de las BDZ por parte de médicos generales y de familia, como psiquiatras y psicólogos podría resumirse en la siguiente sentencia: "las benzodiacepinas son buenas si se usan correctamente". Esta valoración condicional tiene puntos de contacto con las conclusiones a las que arribara en los años 90 el grupo de trabajo sobre BDZ de la American Psychiatry Association (SALZMAN; SHADER, 2015) y supone: a) el reconocimiento de atributos positivos y negativos en las BDZ (por ejemplo: rápidamente efectivas, no riesgo de suicidios, buenos efectos ansiolíticos, riesgo de intoxicación, problemas de memoria, caídas, potencial adictivo), b) un uso mesurado de las mismas (por períodos acotados, dosis flexibles, con plan de retiro) y c) actores que deben vigilar sus propios comportamientos (médicos y pacientes).

Específicamente supone un desplazamiento de la valoración de la propia sustancia hacia la valoración del uso que se hace de ella y de este uso a la valoración de los comportamientos de los individuos implicados. Por consiguiente, la sustancia en sí misma no sería catalogada ni como buena ni como mala, sino que 
serían buenos o malos los comportamientos de los individuos. En este sentido, la responsabilidad de un buen o mal uso recae sobre los individuos y son ellos los que deben orientar moralmente sus comportamientos. Así, para los médicos, la decisión de la prescripción de BDZ, su mantenimiento o retiro, se convierte en un dilema moral al que se debe responder de forma individual. Este dilema se complejiza aún más cuando se describen toda una serie situaciones clínicas que hacen que el uso mesurado se convierta en desmesurado (gravedad del paciente que vuelve la prescripción inevitable, la resistencia de los pacientes a otros tratamientos, presión de los pacientes y terceros, entre otras).

En estas situaciones clínicas, la responsabilidad se vuelca hacia los propios pacientes cuyos comportamientos al ser valorados también moralmente constituyen la base para una tipificación crítica de los mismos (pacientes adictos, pacientes que poseen conocimientos equivocados sobre los medicamentos, pacientes que no obedecen las indicaciones de los profesionales, entre otras). La valoración "neutral" que los profesionales realizan sobre las BDZ coincide con la noción de neutralidad tecnológica que autores como Langdon Winner (1992) ha identificado como sustancial a una visión tradicional, determinista y autónoma de la tecnología.

Así, la tecnología sería entendida como un mero instrumento técnico imparcial y objetivo, dotado de eficiencia interna como medio para conseguir sus fines y sujeto a diversas interferencias externas responsables de los desvíos en su funcionamiento (GONZÁLEZ GARCÍA; LÓPEZ CEREZO; LUJÁN LÓPEZ, 2004). Por consiguiente, la noción de la neutralidad de las BDZ, libera de responsabilidades al propio medicamento y a sus productores, traduce una controversia de colectivos académicos en términos individuales y empaña una posible reflexión sobre una política de medicamentos y el rol de otros actores clave como los laboratorios farmacéuticos o los sistemas regulatorios.

\section{Referencias}

AIBAR, E. Controversias tecnocientíficas públicas: la pericia no es siempre suficiente. Digithum, n. 4, 2002. Disponible en: <http://www.uoc.edu/humfil/articles/esp/aibar0302/ aibar0302_imp.html>. Acceso en: 10 apr. 2016.

BALON, R. Benzodiazepines revisited. Psychotherapy and Psychosomatics, v. 82, n. 6, p. 353-354, 2013.

BOUSSARD, M. et al. Pautas terapéuticas de los trastornos de ansiedad. Revista de Psiquiatría del Uruguay, Montevideo, v. 66, n. 22, p. 130-152, 2002. 
BRANTE, T.; ELZINGA, A. Towards a theory of scientific controversies. Science \& Technology Studies, n. 2, p. 33-46, 1990.

BREGGIN, P. Analysis of adverse behavioral effects of benzodiazepines with a discussion on drawing scientific conclusions from the FDA's spontaneous reporting system. The Journal of Mind and Behavior, Maine, v. 19, n. 1, p. 21-50, 1998.

BURY, M. Caveat venditor: social dimensions of a medical controversy. In: HEALY, D.; DOOGAN, D. P. (Eds.). Psychotropic Drug Development. London: Chapman \& Hall, 1996, p. 41-57.

BUSTELO, P. Estudio de la prevalencia del consumo de tranquilizantes menores en la ciudad de Montevideo. San Pablo: OPS, 1994.

DANZA, A.; CRISTIANI, F.; TAMOSIUNAS, G. Riesgos asociados al uso de benzodiacepinas. Archivos de Medicina Interna, Montevideo, v. 21, n. 4, p. 103-107, 2009.

DONOGHUE, J.; LADER, M. Usage of benzodiazepines: a review. International Journal of Psychiatry in Clinical Practice, n. 14, p. 78-87, 2010.

FERRARI FORCADE, A.; ALFONSO TRINDADE, L. Efectos del Ro5-2807 ("Valium”) sobre el tono muscular y el tiempo reflejo rotuliano en la hemiplejía. El día médico uruguayo. Montevideo, n. 342, p. 4706-4708, 1963.

FRANCES, A. ¿Somos todos enfermos mentales? Manifiesto contra los abusos de la Psiquiatría. Barcelona: Ariel, 2014.

GALEANO MUÑOZ, J. C.; BEDÓ, T. Primeros ensayos clínicos con un nuevo derivado de la benzodiacepina, el Ro5-2807. El día médico uruguayo, Montevideo, n. 342, p. 37823785, 1961.

GALEANO MUÑOZ, J. Nuevo benzodiacepinico de acción intermedia, el Ro5-4556 (Nobrium). El Día Médico Uruguayo, n. 435, p. 318-320, 1969.

GALEANO MUÑOZ, J.; ULRIKSEN DE VIÑAR, M.; VIÑAR, M. N. Acción del Ro45360 en los trastornos del sueño. El Día Médico Uruguayo, n. 369, p. 4956-4958, 1964.

GASPAR, E. Ansiedad y angustia en la clínica psiquiátrica. In: Facultad de Medicina, Escuela de Graduados (Eds.). Primer curso de perfeccionamiento para psiquiatras del cono sur: actualización terapéutica psicofarmacológica, Montevideo: Universidad de la República, 1978, p. 43-52.

GOLDSTEIN, N. Estudio clínico del preparado Ro4-6270 (Limbitrol) en clínica Geriátrica. El Día Médico Uruguayo, n. 394, 1966.

- Experiencias clínicas con el preparado Ro5-2807 (Valium) en los Síndromes epilépticos, fóbicos y obsesivos. El Día Médico Uruguayo, n. 359, p. 4492-4495, 1963 a.

. Experiencias con Valium y sus indicaciones en clínica somática y psiquiátrica. El

Día Médico Uruguayo, n. 362, p. 4633-4637, 1963 b. 
. Tres años de experiencia con "Nobrium": un nuevo tranquilizante. El Día Médico Uruguayo, n. 440, p. 533-535, 1969.

GONZÁLEZ GARCÍA, M.; LÓPEZ CEREZO, J.; LUJÁN LÓPEZ, J. Las concepciones de la tecnología. Ciencia, tecnología y sustentabilidad, El Escorial, 2004, p. 1-16. Disponible en: <http://www.istas.ccoo.es/escorial04/material/dc06.pdf>. Acceso en: 10 apr. 2016.

HERRERA, J. Psicofarmacología de los estados de ansiedad. Revista de Psiquiatría del Uruguay. Montevideo, v. 43, n. 258, p. 215-232, 1978.

LADER, M. Benzodiazepines revisited: will we ever learn? Addiction. London, v. 106, n. 12, p. 2086-2109, 2011.

. Benzodiazepines: the opium of the masses? Neuroscience, v. 3, n. 2, p. 159-165, 1978. . The rise and fall of the benzodiazepines. In: HEALY, D.; DOOGAN, D. P. (Eds.).

Psychotropic Drug Development. London: Chapman \& Hall, 1996, p. 58-68.

LADER, M.; MORTON, S. Benzodiazepine problems. British Journal of Addiction. London, n. 86, p. 823-828, 1991.

LADER, M.; TYLEE, A.; DONOGHUE, J. Withdrawing Benzodiazepines in Primary Care. CNS Drugs, v. 23, n. 1, p. 19-34, 2009.

MATO, M. et al. Estudio de consumo de benzodiacepinas en la Policlínica Psiquiátrica del Hospital Vilardebó. Revista de Psiquiatría del Uruguay. Montevideo, v. 76 n. 1, p. 25-34, 2012.

MEYER, M. From 'cold' science to 'hot' research: the texture of controversy. Papiers $d u$ recherche du CSI, n. 16, 1996. Disponible en: <http:/www.csi.ensmp.fr/working-papers/ WP/WP_CSI_016.pdf>. Acceso en: 10 apr. 2016.

MURGUÍA, D. Psicofarmacología de la ansiedad. Revista de Psiquiatría del Uruguay. Montevideo, v. 52, n. 309-310, p. 206-212, 1987.

REY, A. Intervención de Aída Rey Álvarez. In: JUNTA NACIONAL DE DROGAS (Ed.) Problemas vinculados al consumo de sustancias adictivas. Tomo I. Montevideo: Presidencia de la República-Universidad de la República. Servicio Central de Bienestar Universitario, 1998, p. 350-356.

REY, J. C. Experiencias con un nuevo derivado de la Benzodiacepina, el Ro4-5360. El día médico uruguayo. Montevideo, n. 371, p. 5032-5034, 1964.

SALZMAN, C.; SHADER, R. I. Not Again. Benzodiazepines Once More Under Attack. Journal of Clinical Psychopharmacology, v. 35, n. 5, p. 493-495, 2015.

SCHÖNBERH, A. Traquillizers and hypnotic-sedatives as a social problem in Finland and Sweden. Nordisk Alkohol \& Narkotikatidskrift, n. 14 (English supplement), p. 17-31, 1997.

SOLAL, J. Los medicamentos psicotrópicos o la dependencia confortable. In: EHRENBERG, A. (Ed.) Individuos bajo influencia. Drogas, alcoholes, medicamentos psicotrópicos. Buenos Aires: Nueva Visión, 1994, p. 191-203. 
SPERANZA, N. et al. Consumo de benzodiacepinas en la población uruguaya: un posible problema de salud pública. Revista Médica del Uruguay, v. 31, n. 2, p. 112-119, 2015.

STAHL, S. Don't ask, don't tell, but benzodiazepines are still the leading treatments for anxiety disorders. Journal of Clinical Psychiatry, n. 63, p. 756-157, 2002.

WINNER, L. Autonomous technology. Technics-out-of control as a theme in political thought. Massachussets: The MIT Press, 1992. 369 p.

\section{Agradecimientos}

Lo expresado en el artículo es consecuencia de la Investigación "Clínica de la ansiedad: el uso de benzodiacepinas en las prácticas médica, psiquiátrica y psicológica en los servicios de salud". La misma contó con el financiamiento del Programa de Proyectos de Investigación y Desarrollo de la Comisión Sectorial de Investigación Científica (CSIC) de la Universidad de la República. Se agradece especialmente a Br. Lauren Predebon, por su colaboración en la traducción del artículo al portugués.

\section{Nota}

${ }^{1}$ A. Bielli elaborou e dirigiu o projeto de pesquisa; desenvolveu o trabalho de campo e análise da pesquisa que se apresenta no artigo; redigiu as seções: Avaliaçôes, argumentações e atitudes atuais dos profissionais da saúde; Problemas emergentes na clínica a partir do uso de BZD e Conclusôes. P. Bacci participou do trabalho de campo e análise da pesquisa apresentada no artigo; redigiu a seção Metodologia. G. Bruno participou do trabalho de campo e análise da pesquisa apresentada no artigo; redigiu a seção Introdução e realizou a correção geral do trabalho. N. Calisto participou do trabalho de campo e análise da pesquisa apresentada no artigo; redigiua seçâo "A discussão sobre as BZD no meio acadêmico uruguaio". S. Navarro participou do trabalho de campo e análise da pesquisa apresentada no artigo; redigiu a seção sobre as controvérsias científicas e a controvérsia sobre BZD. Os autores declaram que náo existe conflito de intereses relacionado com o artigo ou com a pesquisa que lhe deu origem. 


\section{Anexo 1. Pauta de entrevista}

1. Datos personales médicos

Sexo

Edad

Profesión y especializaciones

Lugar de estudio e institución

Años que ejerce

Lugares de ejercicio profesional (Instituciones, ciudades)

Años de ejercicio en el servicio de salud

Nivel de atención en donde ha ejercido en el sector público de salud

Servicios en los que ha ejercido en en el sector público de salud

Vínculo Universidad de la República u otras universidades (si es actualmente docente o lo fue)

Si trabaja en otro lugar no olvidar preguntar la situación allí

2. Ansiedad en la clínica

- ¿Cómo piensa la ansiedad?

- ¿Cuándo piensa usted que la ansiedad se convierte en motivo de consulta?

- ¿Cuál ha sido su experiencia en la práctica clínica con la ansiedad?

- ¿Qué tipos de casos de ansiedad recibe en su consulta? (número en relación a otro tipo de trastornos, características).

- ¿Cómo caracteriza estos cuadros de ansiedad? (presentación, edades, sexo, síntomas asociados, crónicos o agudos)

- ¿Cuáles son las dificultades que encuentra para diagnosticar los trastornos de ansiedad?

- ¿Cuál sería la relación de los cuadros de ansiedad que ve en la clínica con las definiciones de cuadros de ansiedad de los manuales de psicopatología (son iguales, tienen diferencias)?

- ¿Qué lugar piensa usted que ocupa la angustia en los cuadros de ansiedad?

3. Prescripción de BDZ

- ¿Qué lo lleva a recetar a un paciente BDZ por primera vez?

- ¿Qué le genera prescribir BDZ por primera vez?

- ¿Qué consideraciones le transmite al paciente cuando le indica BDZ?

- ¿Qué lo lleva a continuar el tratamiento?

- ¿Cómo actúa en caso de repetir prescripciones recetadas por otros colegas? 
- ¿Cómo ha sido su experiencia al discontinuar el tratamiento con algún paciente?

- ¿Cómo ha sido su experiencia personal en el uso de BDZ? ¿Eso puede influenciar en algo sus hábitos de prescripción?

- ¿Cuáles son las BDZ con las que prefiere trabajar? ¿Por qué? ¿Para qué?

- ¿Cómo evalúa la relación médico paciente mediada por las BDZ?

- ¿Cuál es el retorno que recibe de los pacientes?

- ¿Cuál piensa que es la relación entre las prescripciones de BDZ y factores organizacionales de su trabajo en salud pública?

- Relación con otros tratamientos farmacológicos, ¿qué alternativas hay?

- Relación con tratamientos no farmacológicos, ¿cuáles son las alternativas a las BDZ?

- ¿Cuáles son los tratamientos alternativos a las BDZ en los casos de ansiedad a los que ud. ha recurrido?

\section{Percepción sobre las BDZ}

- ¿Qué diría de las $\mathrm{BDZ}$ en general?

- ¿Cuáles son las ventajas para el paciente que se le prescriban BDZ? (costos, rapidez, escasos efectos secundarios, entre otros)

- ¿Cuáles son las ventajas para el médico del uso de BDZ?

- ¿Qué cree que los pacientes conocen sobre las BDZ?

- ¿Qué piensa de las críticas que se hacen al uso de las BDZ?

- A nivel internacional se han hecho demandas a médicos por dependencia inducida a las BDZ. ¿Qué piensa usted de esto?

- ¿Qué piensa de que las BDZ usualmente se prescriban por períodos mayores a los recomendados?

- ¿Cómo cree que es la situación del uso de BDZ en el país y en el sector público de salud?

- ¿Qué tipo de formación específica ha recibido sobre ansiedad? (cursos en la facultad, cursos ofrecidos por laboratorios, etc.)

- ¿Qué tipo de información específica ha recibido sobre las BDZ? (visitadores médicos)

- ¿Cuál piensa que es el rol de los laboratorios farmacéuticos en la situación actual de uso de BDZ en el país?

- Solicitar ejemplos de casos 
A controvérsia cientifico-técnica sobre as benzodiazepinas entre profissionais de saude pública do Uruguai

A controvérsia científico-técnica internacional sobre as benzodiazepinas, intensa durante os anos 1980 e 1990, questionou seu lugar na prática clínica devido a sua potencialidade aditiva e pelo abuso que médicos e pacientes pareciam realizar. Este artigo apresenta resultados de uma pesquisa que teve como objetivo analisar o papel dessa controvérsia nas práticas médica, psiquiátrica e psicológica nos serviços de saúde pública do Uruguai. Utilizou-se metodologia qualitativa e combinou-se um levantamento de artigos acadêmicos nacionais (1960-2012), entrevistas em profundidade com 45 profissionais e dois grupos de discussão. Realizou-se análise de conteúdo a partir de quatro eixos: ansiedade na clínica, prescripção, relação entre tratamentos farmacológicos e não farmacológicos e avaliaçáo das benzodiacepinas. Obteve-se um panorama diacrônico da controvérsia acadêmica e identificou-se uma avaliação condicional desses medicamentos realizada pelos profissionais, que supóe: reconhecimento de atributos positivos e negativos das benzodiazepinas, uso controlado, médicos e pacientes vigilantes de seus próprios comportamentos. Conclui-se que a controvérsia é levantada em termos individuais, o que obstaculiza uma discussão global das dimensôes políticas e coletivas implicadas.

Palavras-chave: benzodiazepinas; controvérsia científica; serviços de saúde; prescrição; psicofármacos. 


\section{Abstract}

\section{The scientific-technical controversy over benzodiazepines among public health professionals in Uruguay}

The international technoscientific controversy on benzodiazepines, especially intense during the 1980 s and 1990s, questioned the place of benzodiazepines in clinical practice because of its addictive potentiality and the abuse of physicians and patients. This article presents some results from a research that aimed to analyze the role of benzodiazepine controversy in medical, psychiatric and psychological practices in Uruguayan public health services. This research methodology was qualitative, combining a review of national academic articles (1960-2012), in-depth interviews with 45 professionals and two discussion groups. Content analysis was carried out using four axes: anxiety in clinic practice, prescription, relationship between pharmacologic and non-pharmacologic treatment, and benzodiazepines valoration. We obtained a diachronic pictures of the academic controversy and we identified a conditional assessment of these medicines made by the professionals. This assessment implies: the recognition of positive and negative attributes of benzodiazepines, a controlled use of benzodazepines, and professionals and patients that must watch their own behaviors. We conclude that the controversy is presented mainly in individual terms, and this prevents a global discussion on the political and collective dimensions involved.

Keywords: benzodiazepines; scientific controversy; health services; prescription; psychotropic drugs. 